\title{
EDITORIAL OPEN \\ Design and discovery of materials guided by theory and computation
}

\author{
npj Computational Materials (2015) 1, 15007; \\ doi:10.1038/npjcompumats.2015.7; published online \\ 25 November 2015
}

Computational materials science and engineering has emerged as an interdisciplinary subfield spanning materials science and engineering, condensed matter physics, chemistry, mechanics and engineering in general. Modern materials research often requires a close integration of computation and experiments in order to fundamentally understand the materials structures and properties and their relation to synthesis and processing. A number of computational methods and tools at different spatiotemporal scales are now well established, ranging from electronic structure calculations based on density functional theory, ${ }^{1,2}$ atomic molecular dynamics ${ }^{3,4}$ and Monte Carlo techniques, phase-field method ${ }^{6-9}$ to continuum macroscopic approaches. Over the last few years, computational materials activities have been steadily moving from technique development and purely computational studies of materials towards discovering and designing new materials guided by computation, machine learning and data mining or by a closely tied combination of computational predictions and experimental validation. This movement is being further accelerated by the recent initiatives by various government agencies in the United States, Europe, China and other countries to pursue the materials genome initiative, $^{10}$ integrated computational materials engineering ${ }^{11-13}$ as well as the 'Big Data' initiative.

The design of materials guided by computation is expected to lead to the discovery of new materials, reduction of materials development time and cost, and the rapid evolution of new materials into products. ${ }^{14}$ New materials discovered or designed by computation or a combination of computation and experiment can be new materials chemistry such as new combinations of atom species, molecules and point defects. They can be new structures of a material of known chemistry including new crystal structures, new microstructures, new interfacial (interface or surface) structures, or new artificial heterostructures or composites. Materials may also exhibit dramatically enhanced properties under an external potential or field or constraint, e.g., mechanical strain or stress or electric field or magnetic field.

The new journal, npj Computational Materials, aims to publish timely original research or perspective and review articles on the design of materials guided by theory/computation, by a combination of theory/computation and experiment, the validation of the computationally predicted/designed materials by experiments, and/or the fundamental understanding of their structures and properties. The focus is on design and discovery of new materials rather than on purely computational technique improvement or incremental theoretical understanding of materials structures and properties, i.e., the discovery of new materials with desired properties will be the central theme of this journal. All types of materials systems are of interest, including both hard and soft materials used in electronic, photonic, memory, medical, magnetic and energy conversion and storage devices, as well as structural materials in automobile and aerospace applications. Although much of the focus in this field to date has been on crystalline materials, we expect that analogues developments in amorphous materials, or on liquids such as electrolytes or melts, where the lack of order adds significantly to the complexity.

A key component to the success of materials design is the availability of materials data. Materials data can be generated through computation or experiments or a combination of computation and experiments, including high-throughput calculations of materials structures and properties and high throughput, combinatorial experimental measurements. In cases where experimental data are not available or very difficult or impossible to measure, computation is not only desirable but also the only means. For example, nanoscale, metastable precipitates are primarily responsible for the mechanical strengthening of many structural materials. Since many of these metastable precipitates are not thermodynamically stable in isolation, their structural and property data as well as the interfacial energy between them and the matrix can only be obtained through computation. The large sets of data and databases can then be mined and analysed for the discovery of novel materials. The journal is interested in publishing original articles on how materials data are generated or how they could be used in materials design or to guide materials design.

npj Computational Materials will also publish articles on the development of new computational tools that allow or facilitate the prediction/design of new materials including significant advances in high-throughput computational techniques, machine learning and data mining or multiscale tools or integrated computational materials engineering methodology. ${ }^{11-13}$

Understanding of materials is the prerequisite for materials design. The journal therefore also welcomes the submission of manuscripts on the fundamental understanding of the structures and properties of predicted/designed materials through a combination of computation and experiments. The special emphasis will be on experimental verification of the theoretical predication and feedback to theory. For example, the broad range of imaging and spectroscopic tools provide information on materials structure and functionality on macroscopically averaged, mesoscopic and local levels. In particular, atomically resolved imaging techniques based on electron and scanning probe microscopy can provide structural and property information on atomic level, whereas scattering techniques provide similar information in $k$ space. Systematic analysis and curation of these types of data will allow construction of libraries of structure-property relationships at atomic levels, effectively sampling regions in the chemical space of a system. This knowledge can then be incorporated in theoretical models, e.g., by providing realistically observed (as opposed to all theoretically possible) defect configurations and providing refinement of theoretical model parameters.

Finally, the ultimate goal is to synthesize materials of interest. While theoretical methods can suggest candidate materials with required properties and provide insight into their thermodynamic stability, actual synthesis can be affected by many other factors, and for many materials, is more of art than science. For example, recent studies in the field of organic molecule design suggest that for many materials the use of big data and crowdsourcing approaches to tap into collective expert opinions can allow estimating the difficulty of synthesis. Of interest is the extension of these approaches to inorganic materials, using both real-time process monitoring and correlative analysis of materials 
microstructure versus process parameters and development of text analytic methods that will allow mining similar information from published body of work.

In summary, design and discovery of materials guided by theory and computation is an emerging field of materials science. The journal, npj Computational Materials, will provide an international platform for the community to exchange ideas and findings. It will report the most recent discoveries and advances of the field and provide its perspectives for future opportunities and challenges.

Long-Qing Chen ${ }^{1}$, Li-Dong Chen ${ }^{2}$, Sergei V Kalinin ${ }^{3}$, Gerhard Klimeck ${ }^{4}$, Sanat K Kumar ${ }^{5}$, Jörg Neugebauer ${ }^{6}$ and Ichiro Terasaki ${ }^{7}$

${ }^{1}$ Department of Materials Science and Engineering, The Pennsylvania State University, University Park, PA, USA;

${ }^{2}$ Shanghai Institute of Ceramics, Chinese Academy of Sciences, Shanghai, China,

${ }^{3}$ Materials Sciences and Technology Division, Oak Ridge National Laboratory, Oak Ridge, TN, USA

${ }^{4}$ Department of Electrical and Computer Engineering, Purdue University, West Lafayette, Indiana, IN, USA;

${ }^{5}$ Department of Chemical Engineering, Columbia University, New York, NY, USA

${ }^{6}$ Department of Computational Materials Design, Max Planck Institute, Duesseldorf, Germany and

${ }^{7}$ Department of Physics, Nagoya University, Nagoya, Japan Correspondence: L-Q Chen (lac3@psu.edu)

\section{REFERENCES}

1 Hohenberg, P. \& Kohn, W. Inhomogeneous electron gas. Phys. Rev. B 136 864 (1964).

2 Kohn, W. \& Sham, L. J. Self-consistent equations including exchange and correlation effects. Phys. Rev. A 140, 1133 (1965).
3 Alder, B. J. \& Wainwright, T. E. Studies in molecular dynamics. I. General method. J. Chem. Phys. 31, 459-466 (1959).

4 Rahman, A. Correlations in the motion of atoms in liquid argon. Phys. Rev. 136 A405 (1964).

5 Binder, K. The Monte Carlo Method in Condensed Matter Physics (Springer: New York, NY, USA, 1995).

6 Chen, L. Q. Phase-field models for microstructure evolution. Annu. Rev. Mater. Res. 32, 113 (2002).

7 Steinbach, I. Phase-field models in materials science. Model. Simul. Mater. Sci. Eng. 17, 073001 (2009).

8 Boettinger, W. J., Warren, J. A., Beckermann, C. \& Karma, A. Phase-field simulation of solidification. Annu. Rev. Mater. Res. 32, 163 (2002).

9 Provatas, N. \& Elder, K. Phase-Field Methods in Materials Science and Engineering (Wiley-VCH: Weinheim, Germany, 2010).

10 Materials genome initiative for global competitiveness. Executive Office of the President National Science and Technology Council (2011).

11 Committee on Integrated Computational Materials Engineering, National Materials Advisory Board, Division on Engineering and Physical Sciences, National Research Council, Integrated Computational Materials Engineering: A Transformational Discipline for Improved Competitiveness and National Security. National Academies Press (2018).

12 Schmitz, G. J. \& Prahl, U. Integrative Computational Materials Engineering-Concepts and Applications of a Modular Simulation Platform. (Wiley VCH Verlag: Weinheim, Germany, 2009).

13 Horstemeyer, M. F. Integrated Computational Materials Engineering for Metals: Using Multiscale Modeling to Invigorate Engineering Design with Science (Wiley, 2011).

14 Olson, G. Designing a new material world. Science 288, 993 (2000).

(i) This work is licensed under a Creative Commons Attribution 4.0 cc International License. The images or other third party material in this article are included in the article's Creative Commons license, unless indicated otherwise in the credit line; if the material is not included under the Creative Commons license, users will need to obtain permission from the license holder to reproduce the material. To view a copy of this license, visit http://creativecommons.org/licenses/ by/4.0/ 